\title{
Stochastic analysis of two-phase immiscible flow in stratified porous media
}

\author{
VINCENT ARTUS $^{1}$, FREDERICO FURTADO ${ }^{2 *}$, BENOÎT NOETINGER ${ }^{1}$ \\ and FELIPE PEREIRA ${ }^{3}$ \\ ${ }^{1}$ Institut Français du Pétrole, 1 \& 4 Avenue de Bois-Préau \\ 92852 Rueil Malmaison Cedex, France \\ ${ }^{2}$ Department of Applied Mathematics and Statistics, SUNY at Stony Brook \\ Stony Brook, NY 11794-3600, U.S.A. \\ ${ }^{3}$ Instituto Politéctnico da Universidade Estadual do Rio de Janeiro \\ Rua Alberto Rangel s/n, 28630-050 Nova Fiburgo, RJ, Brazil \\ E-mails: vicent.artus@ifp.fr / furtado@uwyo.edu / benoit.noetinger@ifp.fr / \\ pereira@iprj.uerj.br
}

\begin{abstract}
This paper investigates the physics of two-phase, immiscible flow in stratified porous media. A review of previous studies reveals the major role played by the interaction of heterogeneity and viscous forces in the development of large-scale flow regimes. The stabilizing effects of gravity for flow in vertically layered porous media is introduced in a first order theoretical model and illustrated with the results of Monte Carlo simulations.
\end{abstract}

Mathematical subject classification: $76 \mathrm{~S} 05,76 \mathrm{~T} 99$.

Key words: Two-phase flow, heterogeneity, Monte Carlo.

\section{Introduction}

The study of macroscopic field-scale aspects of two-phase, immiscible flow in heterogeneous porous media is a challenging issue, with applications to petroleum engineering and hydrogeology. Although several approaches have been proposed for the coarsening of two-phase flow parameters from highly detailed geological models to scales appropriate for flow simulations (Kyte and

\#589/03. Received: 03/XI/03. Accepted: 17/I/03.

*Permanent address: Department of Mathematics, University of Wyoming, USA. 
Berry, 1975, Barker and Thibeau, 1992, Durlofsky, 1997), the physical mechanisms governing the development of large-scale patterns in such flows have only been partially investigated (Lenormand, 1996 and 1997).

Over the past decade, stochastic analyses based on perturbation expansions have been conducted for the investigation of (mean) large-scale behavior of two-phase flow in heterogeneous media (Langlo and Espedal, 1994, Dagan and Cvetkovic, 1996, Zhang, Li and Tchelepi, 1999). Such analyses hinge on the assumption that the effects of the nonlinear viscous coupling between the saturation and pressure fields can be neglected. Under this assumption, the velocity field and, consequently, its covariance function are stationary (time independent), and classical techniques from linear (tracer) flow theories apply. As a result, these approaches predict a continuous growth of the large-scale mixing zone during displacement, at rates similar to those observed in linear flow. The nonlinearities in the flow equations influence only the mean speed of the front, and do not affect the flow regime.

However, linear stability analyses of two-phase flow in homogeneous media show that the nonlinear viscous coupling clearly impacts the evolution of the saturation map when the front is perturbed: depending on the frontal mobility ratio, the nonlinear coupling induces a "crossflow" that either stabilizes or destabilizes the flow process (Saffman and Taylor 1958, King and Dunayevsky, 1989). In a heterogeneous medium, permeability variations cause velocity fluctuations which perturb the saturation front and interact with the resulting viscous crossflow during displacement. This interaction is likely to produce distinct flow regimes (Lenormand, 1996, Furtado and Pereira, 2002), depending on the relative strength of heterogeneity and viscous crossflow.

In this paper we investigate the interaction between viscous coupling effects and heterogeneity for two-phase flow in stratified porous media. This investigation employs both detailed Monte Carlo simulations and a first order stochastic model. The effects of gravity are introduced in our study, with the gravity acceleration assumed to be parallel to the direction of the layers. We view this simplification as a first step in the investigation of the stabilizing role played by gravity in the evolution of the saturation front. 


\section{Formulation of the problem}

We consider the incompressible flow of a two-phase fluid (oil and water) in a two-dimensional vertical cross-section of a stratified porous medium. The (stratified) absolute permeability field $K$ has a known geostatistics. The distinct layers are assumed to have the same relative permeability functions, $k_{r i}(S)$, and the same constant porosity $\phi$. We neglect the effects of capillarity. The medium is originally saturated with oil. Water is injected at a constant rate $Q$, uniformly along the inlet face $x=0$, and fluid is produced at constant pressure at the outlet face $x=L_{X}$. "No-flow" conditions are imposed at the other two faces, $y=0$ and $y=L_{Y}$. The effects of gravity are neglected for the moment, but will be included later.

The total filtration velocity $u_{i}(x, y, t)$ for each phase $i$ is given by the two-phase extension of Darcy's law:

$$
u_{i}=-\frac{K k_{r i}(S)}{\mu_{i}} \nabla p
$$

where $p(x, y, t)$ is the pressure, $S(x, y, t)$ is the saturation of water, and $\mu_{i}$ is the viscosity of phase $i$. Combining Darcy's law with the conservation laws for the phases leads to the well known pressure and saturation equations:

$$
\begin{aligned}
& \nabla \cdot(K \lambda(S) \nabla p)=0, \\
& \frac{\partial S}{\partial t}+\nabla(f(S) \mathbf{u})=0 .
\end{aligned}
$$

(The constant porosity has been scaled out by a change of the time variable.) Here $\lambda(S)$ is the total mobility and $f(S)$ is the water fractional flow function. These parameters are given as:

$$
\lambda(S)=\frac{k_{r w}(S)}{\mu_{w}}+\frac{k_{r o}(S)}{\mu_{o}}, \quad f(S)=\frac{k_{r w} / \mu_{w}}{\lambda(S)} .
$$

For $K$ constant (homogeneous medium), solutions of the pressure-saturation equations typically display a Buckley-Leverett saturation profile: a saturation shock at $S=S_{f}$ traveling at speed $v=u_{0} f^{\prime}\left(S_{f}\right)$ leading a rarefaction zone where each saturation plane travels at a constant speed $v=u_{0} f^{\prime}(S)$. The value 
of $S_{f}$ depends on the fractional flow function $f(S)$ and $u_{0}$ is the speed of the bulk flow, determined by the imposed boundary conditions.

The stability of this saturation front with respect to small perturbations depends on the frontal mobility ratio:

$$
M_{f}=\frac{\lambda\left(S_{f}\right)}{\lambda(0)} .
$$

This dependence has been derived by several linear stability analyses. Suppose that in a homogeneous medium the front position $x\left(S_{f}, y, t\right)$ is perturbed slightly around its mean position $u_{0} f^{\prime}\left(S_{f}\right) t$ :

$$
x\left(S_{f}, y, t\right)=u_{0} f^{\prime}\left(S_{f}\right) t+\frac{1}{2 \pi} \int_{-\infty}^{+\infty} \delta x_{\alpha}(t) e^{i \alpha y} d \alpha .
$$

A first order analysis of the flow process then shows that the evolution of the perturbation mode $\delta x_{\alpha}$ is governed by the following equation (King and Dunayevsky, 1989):

$$
\frac{d}{d t} \delta x_{\alpha}(t)=|\alpha| \frac{M_{f}-1}{M_{f}+1} c \delta x_{\alpha}(t),
$$

where $c=u_{0} f^{\prime}\left(S_{f}\right)$. From this equation we see that for favorable mobility ratios, $M_{f}<1$, the flow is stable: (small) perturbations of the water front into the oil-saturated zone will be damped because of a crossflow due to viscous forces which favors the propagation of the trailing parts of the front. If $M_{f}>1$, on the contrary, such perturbations are amplified during the displacement process and the front is unstable.

When the permeability field $K(x, y)$ is heterogeneous, the velocity field is perturbed and the front is deformed during the displacement. The evolution of the deformed front is governed by two interacting mechanisms (Artus, Noetinger and Ricard, 2002). One results from velocity fluctuations induced by heterogeneities alone, and can be understood from tracer flow theories in heterogeneous media (Dagan, 1989). The other is the effect of viscous coupling, and can be understood from linear stability analyses in homogeneous media (King and Dunayevsky, 1989). The interplay between these mechanisms is crucial for the development of the large-scale structure of two-phase flow in heterogeneous media, and will be investigated in the next sections. 


\section{Flow regimes in heterogeneous media}

\subsection{Importance of viscous crossflow}

Two-phase flow in stratified media has been investigated for a long time. Two simplified models, each representing an extreme case of the influence of viscous crossflow, have generally been used. In one extreme (Stiles, 1949, Dykstra and Parsons, 1950), the layers are assumed to be noncommunicant. In this case, viscous crossflow between adjacent layers does not occur and the only effect of viscous coupling is to change the velocity in each layer as water is injected into the medium. If the mobility ratio is unfavorable $\left(M_{f}>1\right)$, the mobility in each layer increases as the front advances, and the resultant increase in velocity is larger in the more permeable layers than in the less permeable ones. This enhancement of velocity contrast across distinct layers results in a dramatic reduction of the sweep efficiency of the recovery mechanism. If the mobility ratio is favorable $\left(M_{f}<1\right)$, the velocity contrast across layers is attenuated as the flooding progresses, due to the reduction of fluid mobility, but the flow in more permeable zones remains faster than the flow in less permeable ones.

In the other extreme (Hearn, 1971, Simon and Koederitz, 1982, Yortsos, 1992), perfect communication between adjacent layers is assumed. In this case, referred to as vertical equilibrium, viscous crossflow between layers occurs instantaneously so that the component of the pressure gradient in the direction perpendicular to the layers vanishes. If the mobility ratio is favorable, the effect of this crossflow is to speed up the flow in slower layers resulting in less variability of the front position across the layers. If this crossflow is sufficiently strong, some adjacent layers with distinct permeability values can even be invaded with the same velocity. In these zones, the relative position of the front across layers remains unchanged during flooding. We will further investigate this mechanism later in this section.

A comparison between the models of noncommunicant and perfectly communicant layers (Zapata and Lake, 1981, Fitzmorris, Kelsey and Pande, 1992) reveals in a simple way the major role played by viscous coupling in the evolution of two-phase fronts in stratified media. However, these models consider extreme cases and a more general model should be developed to describe all the physical mechanisms involved.

Comp. Appl. Math., Vol. 23, N. 2-3, 2004 


\subsection{Small heterogeneity contrasts}

Recently (Artus, Noetinger and Ricard, 2002), a first order model for two-phase flow at large scales was developed to include the effects of viscous coupling without the simplifying assumption of vertical equilibrium. This analysis, summarized below, focused on the dynamics of the front position $x\left(S_{f}, y, t\right)$ in heterogeneous media.

At first order, velocity fluctuations can be expressed as the sum of two contributions:

$$
\delta \mathbf{u}(x, y, t)=\delta \mathbf{u}_{a}(x, y, t)+\delta \mathbf{u}_{b}(x, y, t) .
$$

The first contribution arises from velocity changes induced by viscous coupling alone (velocity fluctuations in a homogeneous medium with a perturbed saturation map). The second contribution comprises velocity fluctuations induced by heterogeneities alone (the saturation field is assumed to be a Buckley-Leverett profile).

Decomposing all pertinent variables into mean values plus a sum of perturbation modes,

$$
\begin{aligned}
K(x, y) & =K_{0}(x, y)+\frac{1}{2 \pi} \int_{-\infty}^{+\infty} \delta K_{\alpha}(x) e^{i \alpha y} d \alpha, \\
u_{b x}(x, y, t) & =u_{0}+\frac{1}{2 \pi} \int_{-\infty}^{+\infty} \delta u_{b \alpha x}(x, t) e^{i \alpha y} d \alpha, \\
u_{b y}(x, y, t) & =\frac{1}{2 \pi} \int_{-\infty}^{+\infty} \delta u_{b \alpha y}(x, t) e^{i \alpha y} d \alpha, \\
x\left(S_{f}, y, t\right) & =u_{0} f^{\prime}\left(S_{f}\right) t+\frac{1}{2 \pi} \int_{-\infty}^{+\infty} \delta x_{\alpha}(t) e^{i \alpha y} d \alpha,
\end{aligned}
$$

and substituting into the flow equations (1-2) yields the following equations for 
the evolution of front perturbations in a heterogeneous medium:

$$
\frac{d}{d t} \delta x_{\alpha}(t)=|\alpha| \frac{M_{f}-1}{M_{f}+1} c \delta x_{\alpha}(t)+f^{\prime}\left(S_{f}\right) \delta \mathbf{u}_{b \alpha x}(t),
$$

where $c=u_{0} f^{\prime}\left(S_{f}\right)$. The first term on the right-hand side of (3) results from the crossflow induced by the viscous coupling, and is obtained through a linear stability analysis (Saffman and Taylor, 1958, King and Dunayevsky, 1989). The second one is due to permeability fluctuations alone (Dagan, 1989, Langlo and Espedal, 1994). For stratified reservoirs equation (3) simplifies to

$$
\frac{d}{d t} \delta x_{\alpha}(t)=|\alpha| \frac{M_{f}-1}{M_{f}+1} c \delta x_{\alpha}(t)+c \frac{\delta K_{\alpha}}{K_{0}}
$$

whose solution yields the following expression for the evolution of front perturbations:

$$
\delta x_{\alpha}(t)=\frac{c \delta K_{\alpha}}{|\alpha| A K_{0}}\left(e^{|\alpha| A t}-1\right), \quad A=\frac{M_{f}-1}{M_{f}+1} c .
$$

(5) elucidates the influence of viscous effects. For $M_{f}>1$, perturbations of the front position induced by permeability heterogeneities (second term on the right-hand side) are amplified by the viscous term (first term on the right-hand side) and the flow becomes unstable. For $M_{f}<1$, on the contrary, the front perturbations are dampened and a long-time stationary solution follows. This

stationary solution describes the equilibrium situation which is reached when the viscous crossflow balances the effects of permeability variations. In this case, the front line maintains a constant width during flooding.

However, this analysis presupposes small permeability fluctuations. If sufficiently high permeability contrasts appear in the medium, a frontal mobility ratio merely lower than one may not be enough to generate a crossflow that balances the contrasts. It is then plausible to expect that the simple stability diagram predicted by the first-order model (5) will be modified in the presence of more heterogeneous fields.

\subsection{Interaction of viscous effects and heterogeneity}

For layered media, the vertical equilibrium theory predicts two distinct regimes. When the frontal mobility ratio is favorable enough to compensate for permeability contrasts from one stratum to another, the crossflow from the more permeable 
layers to the less permeable ones is such that the front travels at the same speed in all layers. As a consequence, the relative front position across the layers remains stationary and the size of the mixing zone is constant during flooding. Loggia, 1996, has shown that under the vertical equilibrium assumption such "stationary front" occurs as soon as the mobility ratio is greater than the permeability contrasts existing in the medium.

For example, consider a medium with two layers of absolute permeability $K_{1}$ and $K_{2}$ and assume that $K_{1}>K_{2}$. The front line travels with velocities $U_{1}$ in the first layer and $U_{2}$ in the second layer. Under the vertical equilibrium assumption, Loggia, 1996 shows that:

$$
U_{1}=\frac{U_{2} K_{1}}{K_{2}} M_{f}
$$

It is clear from this equation that as $M_{f}$ decreases, $U_{1}$ approaches $U_{2}$, being exactly to $U_{2}$ equal when $M_{f}=K_{2} / K_{1}$. Below this critical vale for $\mathrm{M}_{f}$, the front will travel at the same speed in the two layers and will be stationary. This derivation can be extended to media with more layers. It shows that when the mobility ratio is low enough, the induced viscous crossflow is sufficient to compensate for permeability contrasts and generates a stationary front.

In media with multiple layers, when the mobility ratio is not sufficient to completely stabilize the flow on the whole width, the front is stationary by parts in the medium, in zones where contrasts are lower than the mobility ratio. In these zones, the front is locally stabilized and travels at different but constant speeds, and the "mixing length" evaluated across the whole width of the medium grows linearly with time. The overall process is hence unstable with a linear front growth that can be easily modeled with an appropriate fractional flow term in the saturation equation (Hearn, 1971, Simon and Koederitz, 1982, Loggia, 1996).

The vertical equilibrium theory, which hinges on the idealized situation of perfect communication between adjacent layers (maximum viscous crossflow), only predicts the following two flow regimes: If the stationary behavior is ensured throughout the whole width of the medium, the mixing length does not grow and the flow is stale; otherwise, the mixing length grows linearly in time and the flow is unstable. In more general cases, as communication is not perfect, the interaction between crossflow and heterogeneity is likely to complicate the stability diagram. 
In previous papers, Furtado and Pereira have investigated the evolution of the front in isotropic fractal heterogeneous media for different values of the mobility ratio and of the heterogeneity index (Furtado and Pereira, 1998 and 2002). Their analysis, based on Monte Carlo simulations, focuses on the mixing length, $M_{L}$, defined as:

$$
M_{L r}(t)=\frac{1}{\left(s_{-}-s_{+}\right)} \int\left|\bar{S}_{r}(x, t)-S_{H}(x, t)\right| d x, \quad M_{L}(t)=\left\langle M_{L r}(t)\right\rangle
$$

Here, $\bar{S}_{r}$ is the average in the direction transverse to the mean flow of the saturation solution corresponding to the realization $K_{r}$ of the permeability field; $S_{H}$ is the homogeneous saturation solution corresponding to the constant permeability $K_{H}=\langle K\rangle ; s_{-}$(respectively $s_{+}$) is the saturation value immediately behind (respectively ahead) the saturation front (in our case, $s_{-}=S_{f}, s_{+}=0$ ).

The results of their analysis show that the interaction between viscous effects and heterogeneity contrasts leads to different flow regimes, depending on the relative strength of nonlinear viscous effects or linear heterogeneous effects. The signatures of these different regimes at large scale are different evolutions of the mixing length with time. When the frontal mobility ratio is close to 1 , the effects from the heterogeneity are predominant, and lead to a macrodispersive behavior for the front. The flow regime is hence essentially linear. When nonlinearity due to viscous effects dominates the flow, a nonlinear stable regime or a nonlinear unstable regime occurs, depending on the value of the mobility ratio at the front $\left(M_{f}<1\right.$ or $\left.M_{f}>1\right)$.

For small heterogeneity index, their analysis agrees with the results of the firstorder analysis. As soon as permeability contrasts are higher, a higher mobility ratio is needed to reach stability. This is in accord with what we have already seen in stratified media under the vertical equilibrium assumption (Loggia, 1996).

In the next sections we will start such an analysis for stochastic layered fields, in order to define the different flow regimes and evaluate the limits of stable regimes. The existence of stable flows is of particular interest for large-scale flow simulations, as in these cases the medium can be considered as a single hydrodynamic flow unit and easily coarsened. In real situations, stable processes are likely to happen: although the end-points mobility ratios between water and oil are often unfavorable, the frontal mobility ratio is often favorable, due to 
the particular shapes of the relative permeability curves. For example, for the quadratic relative permeability curves employed in this study,

$$
k_{r w}(S)=S^{2}, \quad k_{r o}(S)=(1-S)^{2},
$$

the mobility ratio is favorable, $M_{f}<1$, if $\mu_{o} / \mu_{w}<3$.

For many realistic relative permeability curves, the mobility ratio evaluated across the front is still favorable even for viscosity ratios of several decades! This makes waterfloods stable for a lot of application cases. Moreover, this stability due to $k r(S)$ curves is amplified by the stabilizing effect of gravity, as will be shown in the next sections.

\section{Stabilizing effect of gravity for flow in vertical layers}

Our goal is to investigate the impact of gravity on the conditions that define the distinct flow regimes. As in practical cases gravity plays a stabilizing role, we will only focus on flows where the displacing fluid is denser than the fluid in place. For simplicity, we will also assume that layers are vertical, so that the direction of gravity is parallel to the flow. Although such a vertical displacement is more likely to occur during a laboratory experiment than in a realistic geological context, the present study sheds some light on the influence of gravity. We leave the study of dipped layers for future investigations.

Under the conditions above, gravity effects modify the fractional flow function as follows:

$$
f(S)=\frac{\mu_{o} / k_{r o}(S)+K\left(\rho_{w}-\rho_{o}\right) g / u_{0}}{\mu_{w} / k_{r w}(S)+\mu_{o} / k_{r o}(S)} .
$$

Here, $\rho_{i}$ are the phase densities and $u_{0}$ is the flow velocity determined by the injection rate. Since gravity effects modify the fractional flow function, the front saturation $S_{f}$ (figure 1 ) as well as the mobility profiles also change. The magnitude of such changes depends on the permeability and the injection velocity $u_{0}$.

Let us consider a vertical displacement in a homogeneous medium. The extension of the previously discussed linear stability analyses, based on the assumption of piston-like displacements, to include gravity effects yields the following equation for the evolution of a perturbation mode of the front position:

$$
\frac{d}{d t} \delta x_{\alpha}(t)=|\alpha| \frac{M_{f}-1}{M_{f}+1}\left(c-u_{c}\right) \delta x_{\alpha}(t),
$$

Comp. Appl. Math., Vol. 23, N. 2-3, 2004 


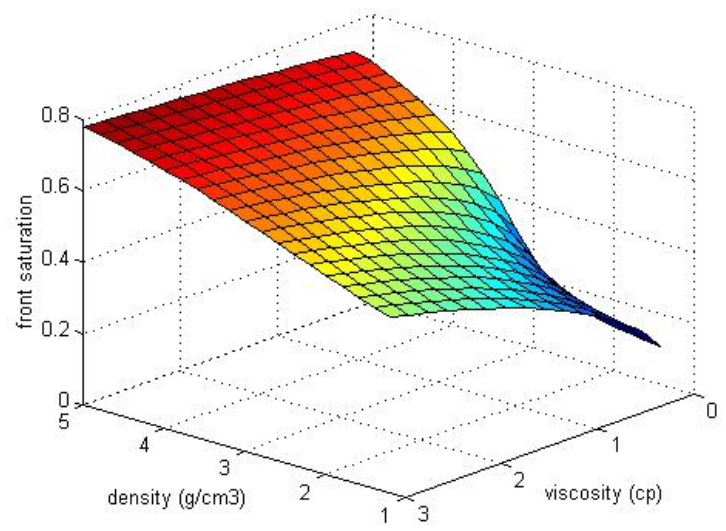

Figure 1 - Evolution of the front saturation value, depending on the injected viscosity $\mu_{w}$ and density $\rho_{w}$, for $Q=0.1 \mathrm{PVI} / \mathrm{year}, \rho o=1.0 \mathrm{~g} / \mathrm{cm}^{3}$ and $\mu o=10 \mathrm{cp}$.

where the critical speed, $u_{c}$, is

$$
u_{c}=\frac{K\left(\rho_{w}-\rho_{o}\right) g}{\lambda^{-1}(0)-\lambda^{-1}\left(S_{f}\right)^{-1}}=\frac{\lambda\left(S_{f}\right) K g \Delta \rho}{M_{f}-1} .
$$

Therefore, if the effects of heterogeneity are also considered, the first order model (4) becomes

$$
\frac{d}{d t} \delta x_{\alpha}(t)=|\alpha| \frac{M_{f}-1}{M_{f}+1}\left(c-u_{c}\right) \delta x_{\alpha}(t)+c \frac{\delta K_{\alpha}}{K_{0}}
$$

where $u_{c}$ is evaluated using the mean permeability $K_{0}$.

We see from (6) that the inclusion of gravity enhances the stability of flows in heterogeneous media. Since we only consider flows where $\Delta \rho>0$, two regimes are possible, according to the sign of:

$$
A_{g}=\frac{M_{f}-1}{M_{f}+1}\left(c-u_{c}\right)
$$

If $M_{f}<1, u_{c}<0$ (since $\Delta \rho>0$ ), $A_{g}<0$ and the flow is stable for any injection rate (recall that we consider flows where water displaces oil, so $c>0$ ). If $M_{f}>1, u_{c}>0$ and the stabilizing effects of gravity mitigate the destabilizing viscous effects. As a consequence, the flow is stable for injection rates such that 
the front speed $c$ is smaller than the critical speed $u_{c}\left(A_{g}<0\right)$. The critical speed depends on the frontal mobility ratio, so it also depends on the injection rate.

This analysis yields the following stability diagram. For a given injection rate $Q$, oil density $\rho_{o}$ and oil viscosity $\mu_{o}$, stable flows will occur in a certain region of the $\left(\rho_{w}, \mu_{w}\right)$ parameter space. This region corresponds to those values of $\left(\rho_{w}, \mu_{w}\right)$ for which the front speed is smaller than the critical speed (figure 2). As the injection rate increases, the size of the stability region decreases, since an increase in the injection rate enhances the destabilizing viscous effects relative to the stabilizing gravity effects (figures 2 and 3 ).

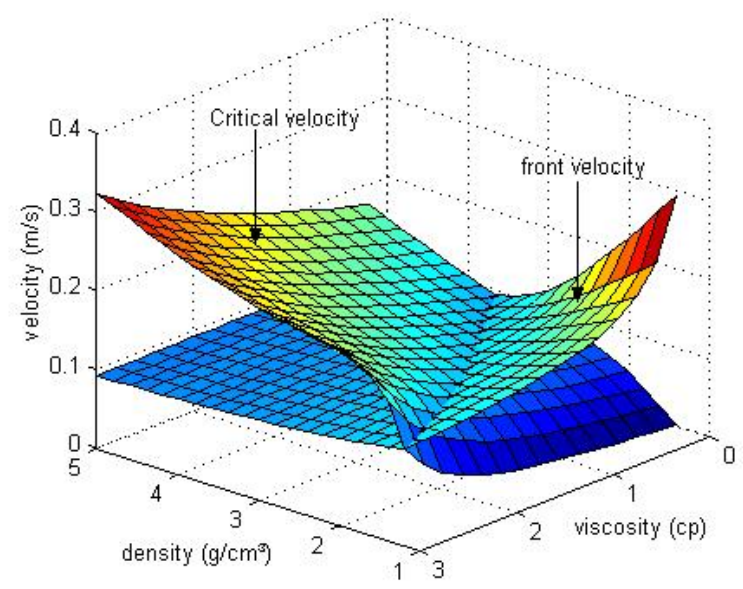

Figure 2 - Evolution of the front velocity and the critical velocity, depending on $\mu_{w}$ and $\rho_{w}$, with $\rho o=1.0 \mathrm{~g} / \mathrm{cm}^{3}$ and $\mu o=10 \mathrm{cp}$, for $Q=0.1 \mathrm{PVI} / \mathrm{year}$. The stable region corresponds to a front velocity lower than the critical velocity.

\section{Numerical experiments}

\subsection{Experiments}

The model (6) is only expected to be valid for slightly heterogeneous media. If sufficiently high contrasts exist in the medium, the viscosity and density of injected fluids have to be increased in order to guarantee stability, as predicted by the vertical equilibrium theory. To study these effects, we performed nu- 


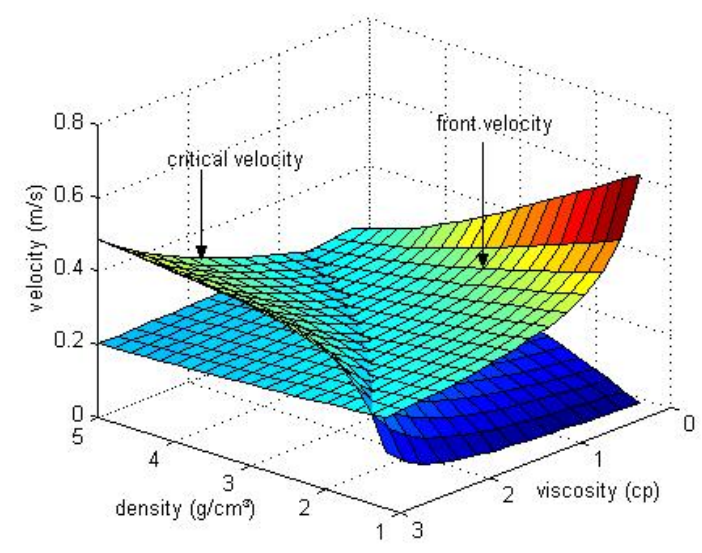

Figure 3 - Evolution of the front velocity and the critical velocity, depending on $\mu_{w}$ and $\rho_{w}$, with $\rho o=1.0 \mathrm{~g} / \mathrm{cm}^{3}$ and $\mu o=10 \mathrm{cp}$, for $Q=0.2 \mathrm{PVI} /$ year.

merical flow simulations in stochastic heterogeneous media, and investigated the appearance of distinct flow regimes as the mobility ratio, injection rate, and density ratio were varied.

Simulations were performed on layered permeability fields. In all simulations, the flow domain was a rectangle $L_{X} \times L_{Y}$ with dimensions $L_{X}=300 \mathrm{~m}$, $L_{Y}=25 \mathrm{~m}$, and a $600 \times 75$ computational grid was used. The permeability field was taken to be lognormal, with mean $K_{0}=100 \mathrm{mD}$, variance $\sigma_{\ln (K)}^{2}=0.1$, and with a Gaussian variogram of correlation length $l_{c}=6 \mathrm{~m}$. Twenty realizations of the permeability field where generated using the FFT-MA algorithm. Relative permeability curves were uniform in the medium: $k_{r w}(S)=S^{2}$ and $k_{r o}(S)=(1-S)^{2}$. Two-phase, immiscible flow was simulated in all fields, using $\rho_{o}=1 \mathrm{~g} / \mathrm{cm}^{3}, \mu_{o}=10 \mathrm{cp}, \rho_{w}$ ranging from 1 to $10, \mu_{w}$ ranging from 1 to 30 and $u_{0}=0.1$ and $0.2 \mathrm{PVI} /$ year. This choice of parameter values allows the investigation of the relative influence of the relevant physical mechanisms and the occurrence of distinct flow regimes.

We employed an IMPES method (implicit in pressure and explicit in saturation), which combines a hybridized mixed finite element method for the solution of the pressure equation and a second-order, nonoscillatory central finite difference scheme for the solution of the saturation equation (Zhu, 2001). 


\subsection{Results}

In order to characterize the flow regimes corresponding to different choices of $\rho_{w}, \mu_{w}$ and $u_{0}$, two measures of the extent of the mixing zone were employed. One is the mixing length $M_{L}$ as defined by Furtado and Pereira. The other is the average distance $L$ between the two extreme points (most and least advanced) of the $2 \mathrm{D}$ front line. Both measures led to the same qualitative results.

Three regimes were observed from our experiments. When viscous and gravity effects are stabilizing enough to compensate for permeability contrasts, the flow is stable and the average front length reaches a fixed equilibrium length (figure 5). It corresponds to a front line completely stationary in the medium. When the stabilizing effects are not strong enough, the average front length grows linearly with time (figure 4 and 8 ). This corresponds to a front line which is continuously stretched during flooding and the flow is unstable. The last regime can be seen as a critical one: viscous and gravity effects do not completely stabilize the front before the breakthrough, and the average front length grows logarithmically during flooding.

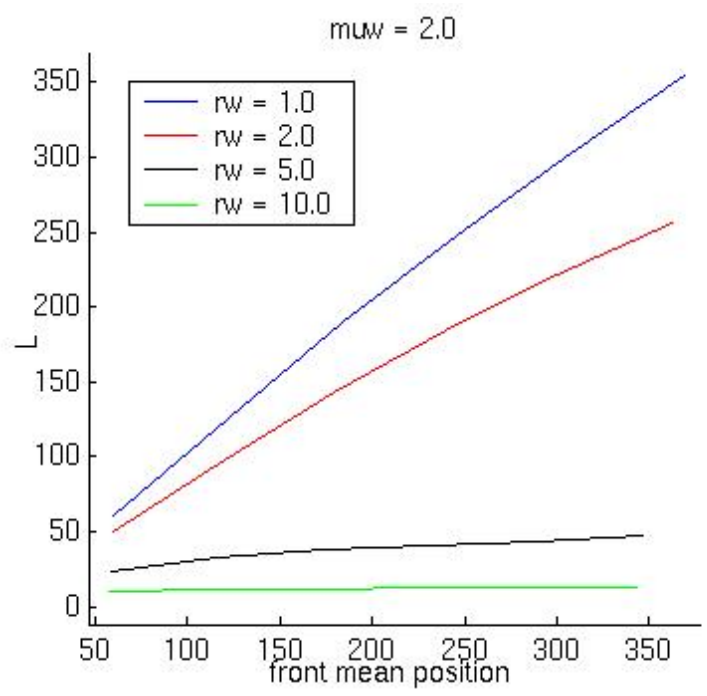

Figure 4 - Evolution of the length of the front $L(\mathrm{~m})$ with traveled distance, depending on the injected density $\rho_{w}$, for $\mu w=2.0 \mathrm{cp}$ and $Q=0.1 \mathrm{PVI} / \mathrm{year}$, with $\rho_{o}=1.0 \mathrm{~g} / \mathrm{cm}^{3}$, $\mu_{o}=10.0 \mathrm{cp}$. 


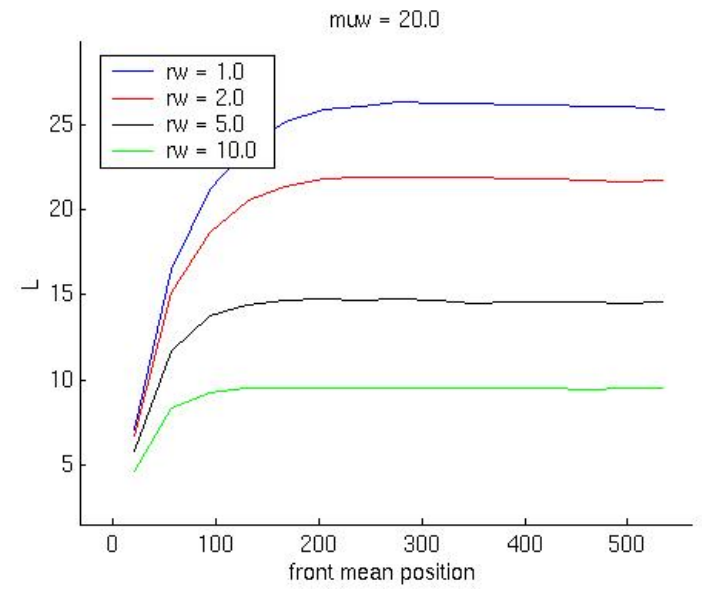

Figure 5 - Evolution of the length of the front $L$ with traveled distance, depending on the injected density $\rho_{w}$, for $\mu w=20.0 \mathrm{cp}$ and $Q=0.1 \mathrm{PVI} / \mathrm{year}$, with $\rho_{o}=1.0 \mathrm{~g} / \mathrm{cm}^{3}$, $\mu_{o}=10.0 \mathrm{cp}$.

Although the first order model predicted that most of our simulations would be stable for a low injection rate $Q=0.1 \mathrm{PVI} /$ year (figure 2), the results of our Monte Carlo simulations show that the stability region is narrower, and stability is reached only for higher viscosity and density values (figures 4 to 8 ). This can be explained by the occurrence of important permeability contrasts in our realizations. When two zones in the medium have very different permeabilities, the viscous crossflow must be very strong to compensate for this contrast and the mobility ratio must be lower, compared to the one predicted by the first order approximation. For example, let us consider the case where gravity has no influence, $\rho_{w}=1.0 \mathrm{~g} / \mathrm{cm}^{3}$. Our first order analysis predicts that the front is stable if $M_{f}<1$, i.e., if $\mu_{w}>3.3 \mathrm{cp}$. Actually, since strong permeability contrasts exist, we only observed stabilization for higher values of the mobility ratio (figures 5 and 7).

Gravity has a clear stabilizing role in our experiments, even for very low mobility ratios, as can be shown from figure 4. As gravity quickly dampens fingers, the mixing length reached after stabilization is much smaller than in flows without gravity effects, even when the latter are stable (figures 4). Stabilized profiles are also reached faster when gravity is included. 


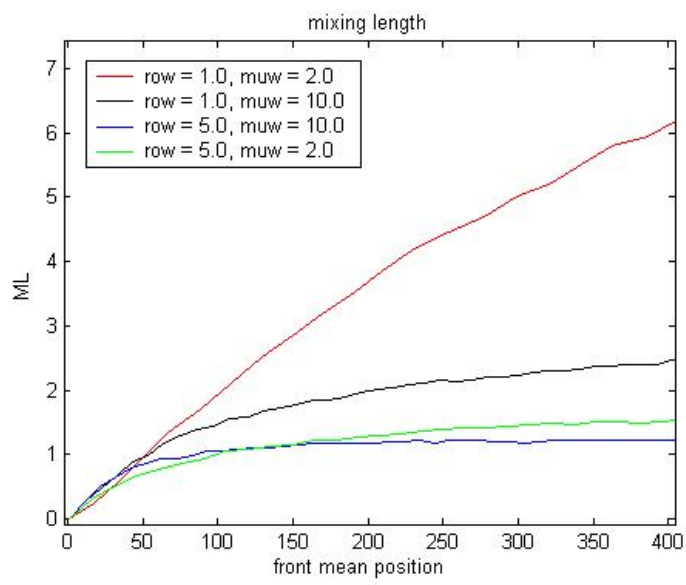

Figure 6 - Evolution of the mixing length $M_{L}$ with traveled distance, depending on the injected density $\rho_{w}$ and viscosity $\mu w=2.0 \mathrm{cp}$, with $Q=0.1 \mathrm{PVI} / \mathrm{year}, \rho_{o}=1.0 \mathrm{~g} / \mathrm{cm}^{3}$ and $\mu_{o}=10.0 \mathrm{cp}$.

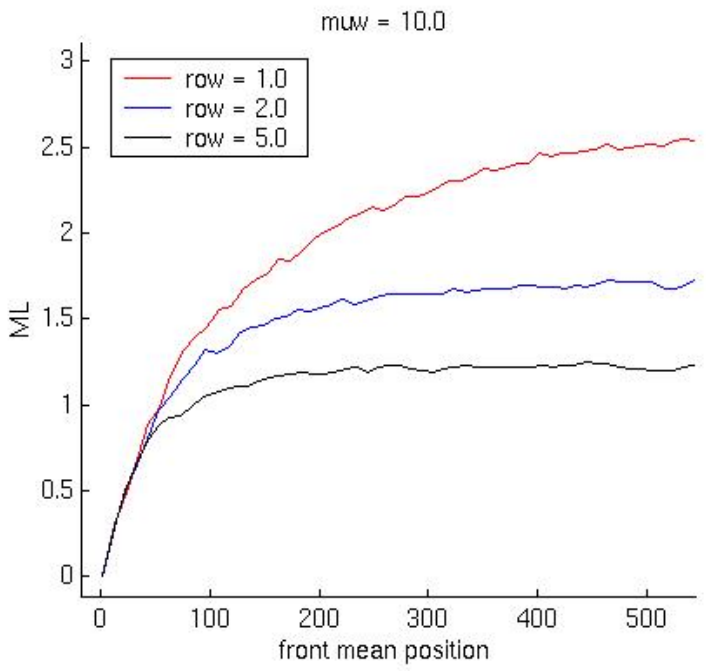

Figure 7 - Evolution of the mixing length $M_{L}$ with traveled distance, depending on the injected density $\rho_{w}$, for $\mu w=10.0 \mathrm{cp}$ and $Q=0.1 \mathrm{PVI} / \mathrm{year}$, with $\rho_{o}=1.0 \mathrm{~g} / \mathrm{cm}^{3}$, $\mu_{o}=10.0 \mathrm{cp}$. 
As predicted by the first-order model, an increase of the injection rate is destabilizing. From figure 8, we can see that when the injection rate is doubled, the growth rate of the front increases, albeit not dramatically. However, this effect is sufficient to trigger the instability of the flow: for the case $\rho_{w}=5.0 \mathrm{~g} / \mathrm{cm}^{3}$ and $\mu_{w}=5.0 \mathrm{cp}$, the flow was stable for $Q=0.1 \mathrm{PVI} /$ year, and unstable for $Q=0.2 \mathrm{PVI} /$ year.

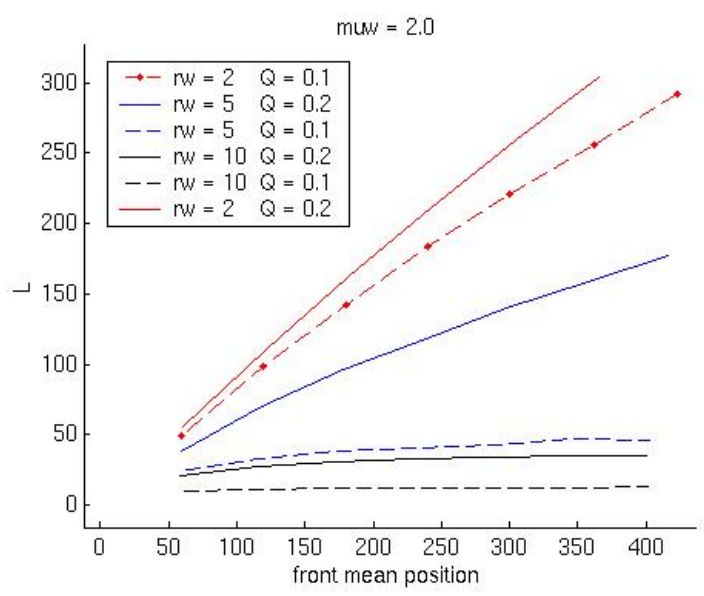

Figure 8 - Evolution of the front length $L$ with traveled distance, depending on the injected density $\rho_{w}$ and viscosity $\mu w=10.0 \mathrm{cp}$ for $Q=0.1 \mathrm{PVI} / \mathrm{year}$ and $Q=0.2 \mathrm{PVI} /$ year.

\section{Conclusions}

We started an investigation of large-scale regimes for two-phase, immiscible flow in vertically stratified reservoirs. The interaction of viscous and gravity effects with heterogeneity is shown to produce different flow regimes, depending on their relative strength.

Gravity is shown to be strongly stabilizing in our experiments: realistic flows in stratified media are then likely to be stable and stationary. In this case, the mixing length reaches an equilibrium value and does not change while flooding. When high permeability contrasts exist in the medium, the front cannot be stationary on the whole width and the mixing length grows linearly with travel distance. 
A first-order model, applicable in the case of small heterogeneity contrasts, was proposed. This model yields the prediction of flow regimes in terms of the front evolution. When the variance of the heterogeneity is higher, the vertical equilibrium theory can predict the flow regime, depending on the value of the mobility ratio across the front, $M_{f}$. The evolution of the stabilizing values for $M_{f}$ and $\Delta \rho$ when the variance of the heterogeneity increases, in a stochastic context, remains to be investigated, as well as the emergence of different flow regimes.

Finally, a stochastic study of stratified fields with dipped layers remains to be performed. Under these conditions, the role of gravity is more complex due to a transverse influence of gravity. Well-developed fingers could then tend to flow downward, and modify the large-scale evolution of the front.

\section{Acknowledgments}

F.P. wishes to acknowledge the support of the Brazilian Council for Development of Science and Technology (CNPq) through Grants 472199/01-3 CTPetro/CNPq, 501886/03-6 CTPetro/CNPq and 470216/2003-4 CNPq. F.F. was supported in part by NSF grant INT-0104529. V.A. work is supported by the IFP and the CNRS. V.A. also acknowledges the Doctoral School "Geosciences et Resources Naturelles" for partially funding his visit to the Instituto Politéctnico in Nova Friburgo. IMPA's high-performance computing cluster was used to produce some of the simulations reported in this work.

\section{REFERENCES}

[1] V. Artus, B. Noetinger and L. Ricard, Dynamics of the water-oil front for two-phase, immiscible flow in heterogeneous porous media. 1-Stratified media. Transport in Porous Media, 56 (2004), 283-303.

[2] J.W. Barker and S. Thibeau, A critical review of the use of pseudo-relative permeabilities for upscaling, SPE paper 35491. European 3-D Reservoir Modelling Conference, Stavanger, Norway, April (1996).

[3] V. Cvetkovic and G. Dagan, Reactive transport and immiscible flow in geological media. II - Applications. Proc. Royal Society of London, 452 (1996), 303-328.

[4] G. Dagan, Flow and transport in porous formations. Springler-Verlag, (1989).

[5] G. Dagan and V. Cvetkovic, Reactive transport and immiscible flow in geological media. I- General Theory. Proc. Royal Society of London, 452 (1996), 285-301.

Comp. Appl. Math., Vol. 23, N. 2-3, 2004 
[6] L.J. Durlofsky, Use of higher moments for the description of upscaled, process independent relative permeabilities. SPE paper 37987, SPE Reservoir Simulation Symposium, Dallas, Texas, June (1997).

[7] H. Dykstra and R.L. Parsons, Secondary recovery of oil in the US, API, (1950).

[8] Y.R. Efendiev and L.J. Durlofsky, Accurate subgrid models for two-phase flow in heterogeneous reservoirs. SPE paper 79680, SPE Reservoir Simulation Symposium, Houston, Texas, 3-5 February (2003).

[9] R.E. Fitzmorris, F.J. Kelsey and K.K. Pande, Effect of crossflow on sweep efficiency in water/oil displacement in heterogeneous reservoirs. Paper SPE 24901. $67^{\text {th }}$ Annual Technical Conference and Exhibition of the SPE, Whashington, W.D.C., 4-7 October (1992).

[10] F. Furtado and F. Pereira, Scaling analysis for two-phase, immiscible flow in heterogeneous porous media. Computational and Applied Mathematics, 17 (3) (1998), 237-263.

[11] F. Furtado and F. Pereira, Crossover from nonlinearity controlled to heterogeneity controlled mixing in two-phase porous media flows. Computational Geosciences, 7 (2003), 115-135.

[12] C.L. Hearn, Simulation of stratified waterflooding by pseudo-relative permeability curves. Journal of Petroleum Technology, p. 805-813, July (1971).

[13] M.J. King and V.A. Dunayevsky, Why waterflood works: a linearized stability analysis. SPE paper 19648, $64^{\text {th }}$ Annual Technical Conference and Exhibition held in Houston, Texas, 16-19 September (1989).

[14] J.R. Kyte and D.W. Berry, New pseudo functions to control numerical dispersion. SPE 5105, pages 269-276, August (1975).

[15] P. Langlo and M. Espedal, Macrodispersion for two-phase, immiscible flow in porous media. Advances in Water Resources, 17 (1994), 297-316.

[16] M. Le Ravalec, B. Noetinger and L.Y. Hu, The FFT moving average (FFT-MA) generator: an efficient numerical method for generating and conditioning Gaussian simulations. Mathematical Geology, 32 (6) (2000), 701-723.

[17] R. Lenormand, Determining flow equations from stochastic properties of a permeability field: the MHD model. SPE Journal, p. 179-190, June (1996).

[18] R. Lenormand, Calculation of fractionnal flow for dynamic upscaling. In proceedings from the $20^{\text {th }}$ IEA Collaborative Project on enhanced Oil Recovery, (1999).

[19] D. Loggia, Etude par acoustique des écoulements de fluides miscibles en milieux poreux. Instabilités-hétérogénéités. Thèse de Doctorat de l'Université Paris VII. Spécialité: Science des Matériaux, (1996).

[20] B. Noetinger, V. Artus and L. Ricard, Dynamics of the water-oil front for two-phase, immiscible flow in heterogeneous porous media. 2-Isotropic media. Transport in Porous Media, 56 (2004), 305-328.

Comp. Appl. Math., Vol. 23, N. 2-3, 2004 
[21] Y. Rubin, Stochastic modelling of macrodispersion in heterogeneous porous media. Water Resources Research, 26 (1) (1990), 133-141, January.

[22] P.G. Saffman and G. Taylor, The penetration of a fluid into a porous medium or Hele-Shaw cell containing a more viscous fluid. Proc. Royal Society of London, A245 (1958), 312-329.

[23] A.D. Simon and L.F. Koederitz, An improved method for the determination of pseudo-relative permeability data for stratified systems. SPE paper 10975, Annual Fall Technical Conference and Exhibition, New-Orlean, L.A., September (1982).

[24] W.M. Stiles, Use of permeability distribution in waterflood calculations. Trans. A.I.M.E. p. 9-13, January (1949).

[25] Y.C. Yortsos, Analytical studies for processes at vertical equilibrium. SPE paper 26022, (1992).

[26] V.J. Zapata and L.W. Lake, A theoretical analysis of viscous crossflow. Paper SPE 1011. $56^{\text {th }}$ Annual Fall Technical Conference and Exhibition of the SPE, San Antonio, Texas, 3-7 October (1981).

[27] D. Zhang, L. Li and H.A. Tchelepi, Stochastic formulation for uncertainty assessment of two-phase flow in heterogeneous reservoirs. SPE paper 51930, SPE Reservoir Simulation and Symposium held in Houston, Texas, February (1999).

[28] J. Zhu, A numerical study of stochastic dispersion for two-phase immiscible flow in porous media, M.Sc. Thesis, University of Wyoming, (2001).

Comp. Appl. Math., Vol. 23, N. 2-3, 2004 